\title{
Publisher Correction: Controllable molecular motors engineered from myosin and RNA
}

Tosan Omabegho, Pinar S. Gurel, Clarence Y. Cheng, Laura Y. Kim, Paul V. Ruijgrok, Rhiju Das, Gregory M. Alushin and Zev Bryant

Correction to: Nature Nanotechnology https://doi.org/10.1038/s41565-017-0005-y (2017); published online 6 November 2017. An incorrect Supplementary Information file was originally published. The file has been replaced with the correct one.

Published online: 2 March 2018

https://doi.org/10.1038/s41565-017-0028-4

\section{Author Correction: Ultrahard carbon film from epitaxial two-layer graphene}

Yang Gao, Tengfei Cao, Filippo Cellini, Claire Berger, Walter A. de Heer, Erio Tosatti, Elisa Riedo and Angelo Bongiorno

Correction to: Nature Nanotechnology https://doi.org/10.1038/s41565-017-0023-9, published online 18 December 2017.

In the version of this Article originally published, the second affiliation for Walter A. de Heer had not been included; it should be 'TICNN, Tianjin University, Tianjin, China'. This has now been added and the numbering of subsequent affiliations amended accordingly in all versions of the Article.

\section{Publisher Correction: Factors that make an impact}

Correction to: Nature Nanotechnology https://doi.org/10.1038/s41565-018-0215-y (2018), published online 6 July 2018.

In the final paragraph of the version of this Editorial previously published, the surname of Hagan Bayley was misspelt as 'Baley'; this has now been corrected in the online versions. 